\title{
Phase I Study of a Vaccine Using Recombinant Vaccinia Virus Expressing PSA (rV-PSA) in Patients With Metastatic Androgen-Independent Prostate Cancer
}

\author{
James Gulley, ${ }^{1,2}$ Alice P. Chen, ${ }^{1}$ William Dahut, ${ }^{1}$ Philip M. Arlen, ${ }^{1,2}$ \\ Anne Bastian, ${ }^{1}$ Seth M. Steinberg, ${ }^{3}$ Kwong Tsang, ${ }^{2}$ Dennis Panicali, ${ }^{4}$ \\ Diane Poole, ${ }^{2}$ Jeffrey Schlom, ${ }^{2 *}$ and J. Michael Hamilton ${ }^{1}$ \\ 'Medical Oncology Clinical Research Unit, Center for Cancer Research, National Cancer Institute, \\ National Institutes of Health, Bethesda, Maryland \\ ${ }^{2}$ Laboratory of Tumor Immunology and Biology, Center for Cancer Research, National Cancer Institute, \\ National Institutes of Health, Bethesda, Maryland \\ ${ }^{3}$ Biostatistics and Data Management Section, Center for Cancer Research, National Cancer Institute, \\ National Institutes of Health, Bethesda, Maryland \\ ${ }^{4}$ Therion Biologics Corporation, Cambridge, Massachusetts
}

\begin{abstract}
BACKGROUND. A Phase I trial of recombinant vaccinia prostate specific antigen (rV-PSA) in patients with advanced metastatic prostate cancer was conducted. This report describes 42 patients who were treated with up to three monthly vaccinations.

METHODS. All patients were entered on a dose-escalation phase I study of recombinant vaccinia containing the gene for PSA (rV-PSA). The primary objective of this study was to determine the safety of this vaccine in metastatic androgen-independent prostate cancer patients. A secondary objective was to assess evidence of anti-tumor activity by PSA measurements, radiologic findings, and immunologic methods.

RESULTS. There was no significant treatment-related toxicity apart from erythema, tenderness, and vesicle formation that lasted several days at the site of injection in some patients. There were immunologic responses, in selected patients, as evidenced by an increase in the proportion of PSA-specific T cells after vaccination. Furthermore, we show that these patients' T cells can lyse PSA-expressing tumor cells in vitro.

CONCLUSION. Given the low toxicity profile and the evidence of immunologic activity, we believe future study is warranted with PSA-based vaccines in prostate cancer. New PSA-based vaccines and vaccine strategies are currently being evaluated. Prostate 53: 109-117, 2002.

(C) 2002 Wiley-Liss, Inc.
\end{abstract}

KEY WORDS: $\quad$ PSA; vaccine; prostate cancer; $\mathrm{T}$ cells

\section{INTRODUCTION}

Prostate cancer is the most common noncutaneous malignancy among American men $(189,000$ cases estimated in 2002) and is the second most common cause of cancer death in men (32,200 deaths) [1]. Although more patients are now diagnosed with localized prostate cancer since the advent of PSA
*Correspondence to: Jeffrey Schlom, Laboratory of Tumor Immunology and Biology, Center for Cancer Research, National Cancer Institute, National Institutes of Health, 10 Center Drive, Room 8B09, Bethesda, MD 20892. E-mail: js141c@nih.gov Received 6 December 2001; Accepted 27 April 2002 DOI $10.1002 /$ pros. 10130 
screening, 30-40\% will develop recurrent disease even after definitive therapy with either surgery or radiation. Patients who develop recurrent disease may be treated with androgen-deprivation strategies; however, within several years, most patients will develop androgen-independent prostate cancer (AIPC). Although chemotherapy has been shown to have palliative benefit in this situation, there is no evidence of prolonged survival. Given the sheer numbers of patients with this disease, the large fraction who fail local definitive therapy and, after recurrence, its inexorable progression to AIPC, there clearly is a need for improved treatment strategies for prostate cancer. One such strategy for targeted therapy is the development of an effective vaccine.

The first step in making a vaccine for tumor immunotherapy is to choose the target antigen. Because prostate specific antigen (PSA) is expressed essentially only in prostatic epithelial cells (normal and malignant), and the prostate gland is nonessential, this antigen is an enticing choice. That PSA is secreted and not membrane bound limits its use as a target for humoral immunity but not its use as a target of specific cellular immune system attack. Cells, including tumor cells, present endogenously expressed proteins on their surface in the form of peptide major histocompatibility complex (MHC) complexes. Cytotoxic $\mathrm{T}$ lymphocytes (CTLs) recognize and are activated by specific peptides in the context of the appropriate MHC class I molecule on antigen presenting cells (APC). This activation can in turn lead to killing of tumor targets by the peptide-specific CTLs. CTL activation as well as tumor recognition and killing, thus, are dependent on the MHC class I molecule. One type of class I molecule, HLA-A2, is present in approximately $50 \%$ of the population in the United States; HLA-A2-restricted peptides from PSA have now been identified [2,3]. The use of PSA as a target to elicit tumor-specific T-cell-mediated lysis has been validated in vitro. Wei et al. showed that expression of human PSA in a mouse tumor cell line could elicit a PSA-specific CTL response and cause tumor cell death in vitro. Immunization with these PSA-expressing cells also caused protection to subsequent tumor challenge in vivo [4]. Correale et al. demonstrated in vitro killing of a PSA-peptide-pulsed HLA-A2positive human cell line by a PSA-specific human CTL cell line, and this lysis was blocked by an antibody directed against class I MHC molecules [2]. Subsequently, it has been shown that PSA-specific CTLs could be generated that lyse PSA-expressing prostate cancer cells [3]. By stimulating normal HLA-A2 donor peripheral blood mononuclear cells (PBMCs) with HLA-A2-restricted PSA peptides in the presence of IL-2, CTL lines were generated that specifically killed
PSA-expressing HLA-A2-positive prostate cell lines, HLA-A2-positive cell lines pulsed with PSA peptide and HLA-A2 cells infected with recombinant vaccinia containing the gene for PSA (rV-PSA) [3].

An active tumor vaccine must express the target antigen in a manner as to produce a vigorous, specific immune response to it. One such way is to use viral vectors to express the antigen gene of interest. Vaccinia has many properties that make it a good expression vector. Large amounts of foreign DNA can be inserted and the transgene remains stably expressed, with accurate replication and efficient posttranslational processing. Vaccinia can also infect a large range of cells, and the recombinant proteins are highly immunogenic [5]. A recombinant vaccinia virus expressing PSA (rV-PSA or PROSTVAC) was constructed by insertion of the PSA gene into the viral genome of the Wyeth (NYCBH) strain of vaccinia. Preclinical safety testing performed in male rhesus monkeys with three immunizations given by skin scarification at 4-week intervals revealed no adverse effects, except for local reactions to the vaccination, with a follow-up of 54 weeks [6].

There have been two prior phase I trials with $\mathrm{rV}$ PSA $[7,8]$. These trials showed that this vaccine is well tolerated and can induce PSA-specific immune responses as well as potential clinical activity. Those trials were both conducted on patients with rising PSA levels after local therapy with no radiographic evidence of metastatic disease. In contrast, in the present study, we evaluate the rV-PSA vaccine in patients with metastatic androgen-independent prostate cancer.

\section{MATERIALS AND METHODS}

\section{Patient Selection}

All patients were enrolled onto a single phase I dose-escalation trial, conducted at the National Cancer Institute (NCI) and Georgetown University, which was approved by the institutional review boards of the respective institutions. Patients were required to have metastatic prostatic adenocarcinoma with evidence of progressive disease by either new bone or soft tissue lesions or a rising serum PSA level (three determinations at least 1 week apart) after at least one therapeutic hormonal manipulation. The patients must have recovered from all acute toxicities of prior treatments, and at least 4 weeks must have elapsed since the patient's prior chemotherapy, radiotherapy, surgery, or second-line hormonal therapy. Patients needed to be Eastern Cooperative Oncology Group performance status 0 through 2 and have adequate hematologic, hepatic, and renal function. In addition, patients were required to have normal immunologic 
testing as defined by positive delayed hypersensitivity skin testing (mumps, Candida, and Trichophyton), no evidence of an immunocompromised state as defined by nonreactive HIV testing, no diagnosis of altered immune function, no prior radiotherapy to $>50 \%$ of nodal groups, no prior splenectomy, and no concurrent steroid use. Prior vaccinia exposure (for smallpox vaccination) was required either by physician certification or detection of anti-vaccinia antibodies.

Exclusion criteria were known egg allergy, active cases or history of skin disorders such as eczema, extensive psoriasis, varicella zoster, impetigo, or burns; history of seizures, serious intercurrent illnesses, other noncutaneous malignant process, and close contact with either immunocompromised individuals, those with the above skin conditions, or children younger than 3 years old. All patients gave written informed consent in accordance with federal, state, and institutional guidelines.

\section{Treatment Plan}

The rV-PSA vaccinations were administered to each patient a total of three times at 4-week intervals. Five groups of patients were given escalating doses of $\mathrm{rV}$ PSA. In each group, six patients were evaluable for toxicity for 28 days before entry of patients at the next highest dose level. The first two groups of patients were given the vaccination by dermal scarification (DS); however, because the required volumes of the subsequent dose levels exceeded those deliverable by a bifurcated needle, the remaining dose levels were given subcutaneously (SC). The doses were as follows: group 1 (8 patients), $2.65 \times 10^{5}$ pfu DS; group 2 (7 patients), $2.65 \times 10^{6}$ pfu DS; group 3 (7 patients), $2.65 \times 10^{7}$ pfu SC; group 4 (13 patients), $2.65 \times 10^{8} \mathrm{pfu}$ SC; and group 5 (7 patients), $2.65 \times 10^{8}$ pfu SC plus GM-CSF $(100 \mu \mathrm{g} /$ day $\times 4$ days starting the day before vaccination) SC at the same site as the vaccination. There was a planned six patients per group with 12 at the maximally tolerated dose, with an additional 6 to 10 patients treated with GM-CSF. Because all six patients had to be evaluable for 4 weeks before enrolling additional patients at the next dose level, additional eligible patients were added at the same dose level. If a patient experienced a grade III toxicity, the GM-CSF in subsequent administrations was reduced to $50 \%$ of the previous dose.

The patients were seen monthly for 6 months with weekly laboratory and telephone follow-up for the first 4 weeks. After the first 6 months, patients were followed up every other month until the disease progressed or a new treatment was started. Complete interval histories, physical examinations, blood chemistries, hemograms, and serum PSA levels were obtained. All patients were evaluated for toxicity by the Common Toxicity Criteria (2-18-88 version) and the vaccinia toxicity grading scale (see below). Doselimiting toxicity (DLT) was defined as a grade 3 or greater toxicity (except for nausea, vomiting, or fever). The maximum tolerated dose was defined to be the dose below the one in which two patients experienced DLT.

\section{Vaccinia-Related Toxicity Grading}

Vaccinia-related toxicity grading was as follows: grade 1 , cutaneous reaction extending to not more than $10 \mathrm{~cm}$ from vaccination site; grade 2, cutaneous reaction extending to more than $10 \mathrm{~cm}$ from the vaccination site or any autoinoculation that resolves without sequelae; grade 3, any toxicity between 2 and 4; grade 4, autoinoculation syndrome with sequelae, e.g., postvaccinia encephalitis, vaccinia gangrenosum, eczema gangrenosum, or Steven-Johnson syndrome [9].

\section{Criteria for Response}

Patients without bidimensionally measurable disease were evaluated for response by PSA as follows: complete response (CR) defined as normalization of PSA level for three successive evaluations obtained at 2-week intervals; partial response (PR) defined as a decline in PSA level by greater than $80 \%$ without normalization for three successive biweekly evaluations; stable disease (SD) defined as less than an $80 \%$ decline in PSA level or up to a $50 \%$ increase in PSA level and no evidence of progressive disease; progressive disease (PD) defined as three consecutive increases in PSA level to $>50 \%$ over the minimum value obtained during response. For patients with bidimensionally measurable disease, the response criteria were defined as follows: CR, the complete disappearance of all tumor and normalization of blood tests for at least 4 weeks; PR, a decrease of at least $50 \%$ of the sum of the products of the perpendicular diameters of all measured lesions and the absence of enlargement of any lesion or appearance of new lesions; SD, changes in measurable diseases too small to meet the requirements for PR or PD for at least 12 weeks without worsening of symptoms; and PD, the development of any new lesion or the increase ( $>25 \%)$ of the product of perpendicular diameter in any pretreatment area of measurable disease.

\section{Vaccine Formulation}

rV-PSA, constructed and manufactured by Therion Biologics Corporation (Cambridge, MA), was provided by CTEP, NCI (NSC \#697729). The vaccine is prepared from virus derived from the Wyeth (New York City Board of Health) strain of vaccinia. 
This was selected based on its favorable toxicity profile. The recombinant virus was constructed by insertion of the entire human PSA gene into the viral genome as described [5]. Each vial of vaccine contained $0.3 \mathrm{ml}$ of vaccine at a concentration of $1.17 \times$ $10^{9} \mathrm{pfu} / \mathrm{ml}$. Administration for the first two groups of patients was by means of dermal scarification with a bifurcated needle with 1 or $2 \mu$ of virus diluted appropriately in PBS, respectively, for groups 1 and 2 . The last three groups received subcutaneous injection by means of needle and syringe. A sterile, nonadherent dressing (i.e., Telfa ${ }^{\mathrm{TM}}$ ) was used to cover the site.

\section{Collection of PBMCs}

Blood was collected in heparinized tubes from HLA-A2-positive patients. The mononuclear cell fraction was separated by Ficoll-Hypaque density gradient separation, washed three times, and frozen in $90 \%$ heat-inactivated human $\mathrm{AB}$ serum and $10 \%$ DMSO at $-195^{\circ} \mathrm{C}$ at a concentration of $1 \times 10^{7}$ cells $/ \mathrm{ml}$ until assayed.

\section{ELISPOT}

Cells were thawed and cultured overnight in RPMI1640 complete medium (Life Technologies, Inc., Gaithersburg, MD) at $37^{\circ} \mathrm{C}$ at $5 \% \mathrm{CO}_{2}$ before performing the ELISPOT assay. A modified ELISPOT assay that detects $\gamma$-IFN production was used to determine the CTL precursor frequency to PSA-3 peptide and Flu peptide [mp 58-66] in both pre- and postvaccination PBMCs [10]. Briefly, 96-well millipore HA plates (Millipore Corporation, Bedford, MA) were coated with $100 \mu \mathrm{l} /$ well of capture monoclonal $\mathrm{AB}$ against human $\gamma$-IFN at a concentration of $10 \mu \mathrm{g} / \mathrm{ml}$ for $12 \mathrm{hr}$ at room temperature. Plates were blocked for $30 \mathrm{~min}$ with RPMI 1640 plus $10 \%$ human antibody serum. PBMCs $\left(2 \times 10^{5}\right)$ were added to each well. PSA-3 pulsed C1R-A2 cells were added into each well as APC at an effector:APC ratio of 1:1. Unpulsed C1R-A2 cells were used as a negative control. HLA-A2 binding Flu peptide was used as a positive peptide control [11]. Cells were co-incubated for $24 \mathrm{hr}$ and lysed with phosphate buffered saline (PBS)-Tween $(0.05 \%)$. Biotinylated anti- $\gamma$-IFN antibody diluted to $2 \mu \mathrm{g} / \mathrm{ml}$ in PBS-Tween containing 1\% bovine serum albumin (BSA) was added and incubated overnight in 5\% $\mathrm{CO}_{2}$ at $37^{\circ} \mathrm{C}$. Plates were then washed three times and developed with avidin alkaline phosphatase (GIBCO/ BRL, Grand Island, NY) for $2 \mathrm{hr}$, after which each well was examined for positive dots. The number of dots in each well was counted by two separate investigators in a blinded manner, and the frequency of responding cells was determined for a total of $6 \times 10^{5}$ effector cells plated.

\section{Generation of T-Cell Lines}

The protocol described by Tsang et al. was used for the generation of PSA-specific T-cell lines [12]. Briefly, PBMCs were obtained from the heparinized blood of HLA-A2-positive patients. The mononuclear fraction from each donor was washed three times with AIM V medium (Life Technologies, Inc.) supplemented with $5 \%$ human blood-type AB serum (Valley Biomedical, Winchester, VA), $2 \mathrm{mM}$ glutamine, $100 \mathrm{U} / \mathrm{ml}$ penicillin, and $100 \mu \mathrm{g} / \mathrm{ml}$ streptomycin (Life Technologies, Inc.). The PBMCs were subsequently resuspended in this complete medium. Cells $\left(2 \times 10^{5}\right)$ in a complete volume of $100 \mu \mathrm{l}$ were put into individual wells of a 96well flat-bottom assay plate (Corning Costar Corp., Cambridge, MA). PSA-3 was added to a final concentration of $50 \mu \mathrm{g} / \mathrm{ml}$. The cultures were incubated for 5 days at $37^{\circ} \mathrm{C}$ in a humidified atmosphere containing 5\% $\mathrm{CO}_{2}$. After removal of the peptidecontaining medium, the cultures were supplemented with IL-2 at a concentration of 20 units/ $\mathrm{ml}$ for 11 days, with IL-2-containing medium replenished every 3 days. The 5-day incubation with peptide and 11-day IL-2 supplement constituted one in vitro stimulation (IVS) cycle. Primary cultures were restimulated with specific peptide on day 11 to begin the next IVS cycle. Irradiated (4000 rads), autologous PBMCs $\left(1 \times 10^{6}\right)$ were used as APC.

\section{Anti-PSA Antibody Enzyme-Linked Immunosorbent Assay}

The presence of anti-PSA antibodies in patient serum pre- and postvaccination was analyzed by using an enzyme-linked immunosorbent assay (ELISA). Polyvinyl chloride 96-well microtiter plates (Dynatech Laboratories, Chantilly, VA) were incubated overnight at $4{ }^{\circ} \mathrm{C}$ with a purified preparation of PSA (Vitro Diagnostics, Littleton, $\mathrm{CO}$ ) as well as bovine serum albumin (BSA) or human serum albumin (HSA) at $100 \mathrm{ng} /$ well in $50 \mu \mathrm{l}$ of PBS (pH 7.2). The wells were blocked for $1 \mathrm{hr}$ with PBS containing 5\% BSA and then washed once with PBS containing 1\% BSA (assay buffer). Patient serum and control pooled human serum (Gemini Bioproducts, Calabasas, CA) were diluted in assay buffer and added to wells in triplicate in a volume of $50 \mu \mathrm{l} /$ well. Purified murine anti-PSA immunoglobulin G (IgG) antibody (Fitzgerald Industries, Concord, MA) was used as a positive control for PSA binding. An isotyped matched (MOPC-21) $\operatorname{IgG}_{1}$ antibody (Jackson Immunoresearch, West Grove, PA) was used as a negative control. After incubation overnight at room temperature, the wells were washed four times with assay buffer, and $50 \mu \mathrm{l}$ of a 1:4,000 dilution of peroxidase-conjugated goat anti-human IgG (Kirkegaard \& Perry Laboratories, Gaithersburg, 
MD) were added to each well. A 1:2,000 dilution of peroxidase-conjugated goat anti-mouse IgG (Kirkegaard \& Perry) was used for the PSA antibody controls. After incubation at $37^{\circ} \mathrm{C}$ for $1 \mathrm{hr}$, wells were washed four times with assay buffer, and $100 \mu \mathrm{l}$ each of the chromogen $\mathrm{O}$-phenylenediamine dihydrochloride (Sigma, St. Louis, MO) and hydrogen peroxide were added to each well. After a 10-min incubation in the dark, the reaction was stopped with $25 \mu \mathrm{l}$ of $4 \mathrm{~N}$ $\mathrm{H}_{2} \mathrm{SO}_{4}$. The absorbance of each well was measured at $490 \mathrm{~nm}$ by using an ELISA microplate autoreader (BioTek Instruments, Winooski, VT).

\section{Cytotoxic Assay}

Target cells were incubated with $50 \mu \mathrm{Ci}$ of ${ }^{111}$ indium-labeled oxyquinoline (Medi-Physics, Inc., Arlington, IL) for $15 \mathrm{~min}$ at room temperature. Target cells $\left(0.3 \times 10^{4}\right)$ in $100 \mu \mathrm{l}$ of RPMI- $1640 \mathrm{CM}$ were added to each well of 96-well flat-bottomed assay plates (Corning Costar Corp.). The labeled target cells were incubated with peptides for $60 \mathrm{~min}$ at $37^{\circ} \mathrm{C}$ in $5 \% \mathrm{CO}_{2}$ before effector cells were added. Effector cells were suspended in $100 \mu \mathrm{l}$ of $\mathrm{CM}$ supplemented with $10 \%$ pooled human $\mathrm{AB}$ serum, and added to the target cells. The plates were then incubated at $37^{\circ} \mathrm{C}$ in $5 \%$ $\mathrm{CO}_{2}$ for 4 or $16 \mathrm{hr}$. Supernatant was harvested for gamma counting with harvester frames (Skatron, Inc., Sterling, VA). Determinations were carried out in triplicate, and standard deviations were calculated. Specific lysis was calculated with the use of the following formula (all values in cpm): \% Lysis $=[$ (Observed Release - Spontaneous Release $) \backslash($ Total Release Spontaneous Release)] $\times 100$. Spontaneous release was determined from wells to which $100 \mu \mathrm{l}$ of $\mathrm{CM}$ was added. Total releasable radioactivity was obtained after treatment of targets with $2.5 \%$ Triton X-100.

\section{RESULTS}

Forty-two men with metastatic AIPC with rising PSA levels after androgen withdrawal were treated over a 22-month period. The patients' ages ranged from 44-82 years (median, 66 years) with on-study PSA levels ranging from 2.2 to 3,892 (mean, 423; median, 70). Forty patients had metastatic bony disease alone or with soft tissue disease, and two additional patients had only soft tissue disease. Twenty patients required analgesics for diseaserelated symptoms. No DLT occurred, and a maximum tolerated dose of vaccine was not reached.

The toxicities are shown in Table I. In addition, two grade 4 toxicities were seen in one patient (alkaline phosphatase and bilirubin) at the time of disease progression. All patients who received the dermal scarification had an inoculation site reaction as did
TABLE I. Toxicities Observed With rV-PSA Vaccination*

\begin{tabular}{lcc} 
& Grade 2 & Grade 3 \\
\hline Fatigue & 4 & 1 \\
Liver enzymes & 2 & 0 \\
Fever & 5 & 1 \\
Myalgias & 0 & 1 \\
\hline
\end{tabular}

${ }^{*} \mathrm{rV}$-PSA, recombinant vaccinia containing the gene for prostate specific antigen.

most of those patients who received subcutaneous vaccinations (Table II). The reaction rate was $74 \%$ for the first vaccination, dropping to $37 \%$ then $19 \%$ for the subsequent vaccinations. The decrease in inoculation site reactions over the course of vaccination is consistent with neutralizing anti-vaccinia antibodies.

There were no objective tumor responses, and the mean time to progression of disease was 106 days (range, 28-310 days), with all patients eventually progressing or interpreted as progressing because of removal from study for reasons related to disease. Six of 42 patients (14\%) had stable disease, $30(72 \%)$ had progressive disease, and 6 were not assessed. Of those six not assessed, four removed themselves from the study, one was diagnosed with another cancer, and one had a decrease in performance status. Forty-two patients completed the first vaccine cycle, 38 patients completed the second cycle, and 26 patients completed all three cycles.

Twenty-five patients were HLA-A2 positive. Six patients who were HLA-A2 positive and had two or three vaccinations had adequate material for in vitro quantitation of antigen-specific T-cell or cytotoxicity assays. Five of the patients were analyzed for quantitative changes in their PSA-specific T cells over the course of their vaccinations. This analysis was done by means of ELISPOT assay with change in quantitation of influenza-specific $\mathrm{T}$ cells as control. Although there was no appreciable change in the influenza-specific immune response, three of five patients had a twofold or greater $(2.7$ to $>5.7)$ increase in the number of PSA-specific T cells (Table III). None of the patients had any evidence of anti-PSA antibody formation over the course of the vaccine (data not shown). The ability of these PSA-specific T cells to lyse PSA-bearing tumor cells was evaluated in three of three selected HLA-A2-positive patients (patient number 1, 14, and 15, representing dose levels I and II). PSA-specific CTL cell lines were established as described in the Materials and Methods section. These cell lines were used in a CTL assay with ${ }^{111}$ In-labeled HLA-A2-positive target cells that either expressed PSA (LNCaP) or were pulsed with an HLA-A2- 
TABLE II. Number of Patients Exhibiting Cutaneous Response After Each Vaccination With rV-PSA*

\begin{tabular}{lccccc}
\hline Group & No. of patients & Treatment & Dose 1 & Dose 2 & Dose 3 \\
\hline 1 & 8 & $2.65 \times 10^{5} \mathrm{pfu}$ DS & $8 / 8$ & $2 / 6$ & $2 / 5$ \\
2 & 7 & $2.65 \times 10^{6} \mathrm{pfu}$ DS & $7 / 7$ & $3 / 7$ & $1 / 5$ \\
3 & 7 & $2.65 \times 10^{7} \mathrm{pfu} \mathrm{SC}$ & $5 / 7$ & $4 / 7$ & $1 / 5$ \\
4 & 13 & $2.65 \times 10^{8} \mathrm{pfu} \mathrm{SC}$ & $7 / 13$ & $3 / 12$ & $0 / 8$ \\
5 & 7 & $2.65 \times 10^{8} \mathrm{pfu} \mathrm{SC}$ & $4 / 7$ & $2 / 6$ & $1 / 3$ \\
\multirow{2}{*}{ Total } & 42 & $+100 \mu \mathrm{l} \mathrm{GM-CSF}$ & & & \\
\hline
\end{tabular}

*DS, dermal scarification; SC, subcutaneously; rV-PSA, recombinant vaccinia containing the gene for prostate specific antigen.

restricted PSA peptide (C1R-A2 cells pulsed with PSA$3)$. These cell lines were able to specifically lyse these target cells (Table IV).

\section{DISCUSSION}

There have been two previous phase I trials with rV-PSA. In a group of patients who had failed local therapy with rising PSA levels without radiographic evidence of disease (stage D0 disease), and who ceased hormonal therapy at the beginning, Sanda et al. demonstrated that three monthly injections of $\mathrm{rV}$ PSA were associated with a prolonged interval $(>8$ months) from testosterone restoration to PSA rise in one of six patients studied [8]. This patient had a T3N0M0 tumor; when he failed primary therapy, he was put on an LHRH agonist for 10 months before being put on the vaccine. His PSA level remained undetectable for more than 8 months after three vaccinations of rV-PSA, despite the cessation of the
LHRH therapy and a rise in testosterone levels within 6 weeks of interrupting androgen deprivation. Another patient also showed induction of an anti-PSA IgG antibody response, suggesting that this regimen could induce specific humoral immunity to PSA. In a study of 33 patients with D0 disease, Eder et al. showed that a regimen of $\mathrm{rV}$-PSA given monthly was well tolerated [7]. There were four cohorts of patients with the final cohort of 10 patients, at the highest dose of rV-PSA, given GM-CSF at the site of vaccination. Of these 10 patients, 7 were HLA-A2 positive, allowing quantitation of their PSA-specific T cells by means of ELISPOT assay. This assay revealed that there was a twofold or greater increase in the number of PSAspecific $\mathrm{T}$ cells in five of these patients. This immunologic response appeared to be specific, as there was no change in the quantitation of influenza matrix peptidespecific $\mathrm{T}$ cells over the course of the vaccinations. In addition, there was some indication of possible clinical benefit. Fourteen of 33 patients had stable disease for

TABLE III. Analysis of PSA-SpecificT-Cell Responses Using the ELISPOT Assay*

\begin{tabular}{lclcc}
\hline Patient no. & Dose group & Sample & Flu matrix peptide & PSA-3 \\
\hline 14 & 1 & Prevaccine & $1 / 85,714$ & $<1 / 200,000$ \\
& \multirow{2}{*}{2} & Postvaccine & $1 / 54,545$ & $1 / 35,294$ \\
& \multirow{2}{*}{2} & Prevaccine & $1 / 85,714$ & $1 / 200,000$ \\
27 & & Postvaccine & $1 / 66,667$ & $1 / 75,000$ \\
& \multirow{2}{*}{4} & Prevaccine & $1 / 85,714$ & $<1,200,000$ \\
37 & \multirow{2}{*}{5} & Postvaccine & $1 / 150,000$ & $1 / 60,000$ \\
& \multirow{2}{*}{5} & Prevaccine & $<1 / 200,000$ & $<1 / 200,000$ \\
40 & Postvaccine & $1 / 200,000$ & $1 / 120,000$ \\
& 5 & Prevaccine & $<1 / 200,000$ & $1 / 200,000$ \\
& & Postvaccine & $1 / 150,000$ & $1 / 150,000$ \\
\hline
\end{tabular}

*Five patients were studied for activation of their PBMCs in vitro when co-incubated with an HLA-A2-positive antigen presenting cell (C1RA2) pulsed with an HLA-A2-restricted PSA peptide (PSA-3). The activation was measured by ELISPOT assay, with PBMC samples taken prior to vaccination and after two or three vaccinations. The PSA-specific T-cell precursor frequency and influenza (Flu) matrix peptide precursor frequency are shown. PSA, prostate specific antigen; PBMCs, peripheral blood mononuclear cells. 
TABLE IV. Generation of PSA-Specific Cytolytic T-Cell Lines From PBMCs of Patients Vaccinated With rV-PSA*

\begin{tabular}{lccc}
\hline & \multicolumn{3}{c}{ Target } \\
\cline { 2 - 4 } $\begin{array}{l}\text { T-cell line from } \\
\text { patient no. }\end{array}$ & APC + CAP-1 & APC + PSA-3 & LNCaP \\
\hline 1 & $3.0(2.8)$ & $49.3(4.8)$ & $23.7(1.2)$ \\
14 & $2.4(1.9)$ & $41.1(3.7)$ & $35.6(2.8)$ \\
15 & $16.6(1.2)$ & $72.2(6.1)$ & ND \\
\hline
\end{tabular}

${ }^{*}$ Cytotoxic T-lymphocytes cell lines were derived from three of three selected HLA-A2-positive patients. The patients' cell lines were able to lyse C1RA2 cells pulsed with a PSA peptide (PSA-3) as well as an HLA-A2-positive prostate cancer cell line that expresses PSA (LNCaP) in a 16-hr

${ }^{111}$ In release assay with E:T ratio of 25:1. Results are expressed as the percentage of specific lysis of the mean values of triplicate determinations, with SD in parentheses. PSA, prostate specific antigen; PBMCs, peripheral blood mononuclear cells; rV-PSA, recombinant vaccinia containing the gene for PSA; APC, antigen presenting cells (C1RA2); ND, not determined.

at least 6 months with 9 of those 14 having stable disease for 11-25 months.

In the trial reported here, we show that this vaccine regimen is relatively well tolerated in patients with metastatic androgen-independent prostate cancer. This provides important safety data for the use of live vaccinia-based vaccines in advanced cancer patients who may not have an ideally functioning immune system. In addition, we show that some of these patients can overcome tolerance to self-antigens and mount an immune response to PSA. T-cell lines can be established from $\mathrm{T}$ cells taken from this group of patients, which can specifically lyse PSA-expressing tumor cells in vitro. In addition, these T-cell lines could specifically lyse PSA-peptide-pulsed, HLA-matched cell lines but not irrelevant peptide-pulsed HLA-matched cell lines. Furthermore, this group of patients can mount a PSA-specific immune response with a doubling or greater of the pool of circulating PSAspecific T-cells (Table III); however, the significance of the level of increase of PSA-specific T cells seen in this trial is difficult to interpret at this time. The immune responses seen appear to be inadequate to convey significant tumor regression in this setting. There are two potential interrelated reasons for this finding: the patient population selected with advanced disease and potentially concomitant decrease in immune functional status, and the relative lack of potency of this vaccine regimen compared with what is needed for a clinical response, or both.

Although essentially the same vaccine was used in all trials using the rV-PSA vaccine, the patient population was quite different. All patients on the current trial had overt metastasis, whereas no patient on the other two trials had radiographic evidence of metastasis (see Table III). We also saw a trend in the current trial of patients with a higher on-study PSA level having a faster doubling time and progressing off trial earlier. The median PSA level for those who had SD was 15 (range, 2.2 to 3,892; median time to progression, 193 days; range, 119-310 days), whereas the median PSA level for those with progressive disease was 67.4 (range, 8.5 to 2,665.2; median time to progression, 84 days; range, $28-127$ days). There was a trend toward higher initial PSA levels in patients with $\mathrm{PD}\left(\mathrm{p}_{2}=0.14\right.$ by Wilcoxon rank sum test). There was a weak to moderate negative correlation (Spearman correlation coefficient $-0.37 ; P=0.015$ ) between PSA level on-study and time to progression as seen in Figure 1. Thus, those starting the trial with a higher tumor burden as measured by PSA levels tended to have a somewhat more rapid time to progression on this vaccine regimen than those with a lower initial PSA. This correlation was even stronger among patients receiving GM-CSF $(\mathrm{r}=-0.88 ; P=0.017$, after adjusting for comparison between those who received GM-CSF and those who did not) (Fig. 1). Although variances in the underlying disease state may account for differences in time to progression observed, it may be that this vaccine regimen, especially with GM-CSF, may work best against a small disease volume. It may also be possible that continuation of vaccination for more than the three monthly injections given in this trial may prolong stable disease or possibly cause tumor regression.

Vaccination with rV-PSA with three consecutive monthly inoculations did not produce a measurable humoral response in any of our patients and in only 1 of 33 patients from the Eder trial. This finding decreases the likelihood that there would be any relative decrease in measured PSA level due to antibody binding and clearance.

For substantial, beneficial clinical results to be seen, new more-potent strategies are needed to optimally use targeted therapies such as vaccines. There are several ways to make this vaccine approach more potent, and clinical trials evaluating these have been 


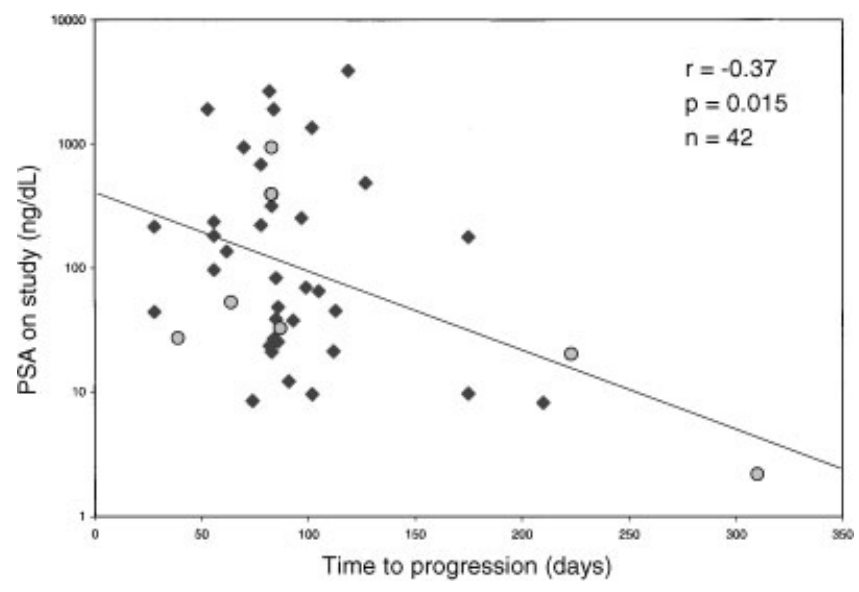

Fig. I. The on-study prostate specific antigen (PSA) serum level correlated with the time to progression as measured by an increase in PSA of $50 \%$ or more or a new lesion. Patients received vaccine with (circles) or without (diamonds) GM-CSF.The Spearman correlation coefficient is -0.37 (weak to moderate correlation, $P=0.015)$.

started recently. These ongoing trials use vaccines and vaccine strategies based on results obtained from preclinical models [13-18]. These are (1) diversified prime and boost vaccinations with different viral vectors encoding the same transgene, (2) T-cell costimulation, and (3) the use of cytokines as adjuvants. These three strategies are briefly discussed below.

A single vaccination with the $\mathrm{rV}$-PSA recombinant at the doses used in this study was shown here to cause the classic vaccinia "take," even in patients with prior smallpox vaccines at childhood. However, further vaccinations with vaccinia are limited by a robust neutralizing response to the vaccinia with a progressively lower response to the transgene after a second or third vaccination. Several investigators have sought alternative strategies for immunization protocols. One such approach is to expose the immune system to the same epitope, but through a different vector, preferably one that can infect cells and produce transgene but cannot replicate, thus minimizing the amount of viral protein seen by the immune system. Recently, several reports have examined this approach in animal models. Murata et al. have shown that alternating immunizations with an influenza virus and a vaccinia virus results in better generation of antigen-specific CD8 $+\mathrm{T}$ cells and cellular immunity than occurs with either virus alone [19]. In studies of tumor treatment, Irvine et al. have reported that alternating vaccinia viruses, avipox viruses, and plasmid DNA gives superior tumor treatment and generation of antigen-specific CTL over single-vector treatment approaches [20]. Hodge et al. [14] and Grosenbach et al. [18] have shown that priming with a vaccinia recombinant and boosting with an avipox recombinant was superior to the use of either vector alone. An ongoing clinical trial is using rV-PSA prime vaccination followed by multiple avipox-PSA boosts. In a phase I clinical trial, Marshall et al. showed that rV-CEA followed by multiple avipox-CEA inoculations (VAAA strategy) in randomized patients was significantly more potent in inducing CEA-specific Tcell precursor frequency than the converse AAAV treatment [21]. In addition, this difference appeared to be clinically relevant, as there was a statistically significant increase in survival in the VAAA arm $(P=0.0018)$. Because avipox does not replicate in humans, this strategy has both safety and the aforementioned immunologic advantages.

Another approach to augment anti-tumor immunity involves T-cell costimulation. A current clinical trial is using priming with rV-PSA admixed with recombinant vaccinia vector containing the B7.1 T-cell costimulatory molecule, followed by multiple boosts with avipox-PSA.

The use of cytokines may also enhance vaccine immunogenicity. GM-CSF, used as a local immunoadjuvant, has been shown to up-regulate class II MHC antigen expression, enhance antigen presentation, and promote dendritic cell maturation and migration to draining lymph nodes [22]. In our trial, the mean time to progression was similar between all dose levels (8695 days), except for the final group that also received GM-CSF (127 days). This finding may have been due to the lower amount of disease in these patients as seen by lower PSA level (median PSA 32.7). However, it may also be due to improved immunostimulation.

There are two phase II clinical trials using these three promising strategies: diversified prime and boost, the use of B7.1 as T-cell costimulation for the priming vaccine, and the use of cytokines (local GMCSF and low-dose IL-2) as immunologic adjuvants $[23,24]$. It is hoped that these advances in potency, in the setting of minimal disease, can lead to improved immunologic and clinical results.

\section{CONCLUSION}

Vaccinia-based vaccine strategies have proven to be safe in patients with advanced prostate cancer and may induce immune responses in those patients. There is growing evidence that supports using vaccines in early, low tumor burden disease, and using more potent vaccine strategies. Second-generation vaccines and vaccine strategies focus on giving vector-driven PSA with costimulatory molecules, using different viral vectors containing the same transgene for priming and boosting (diversified prime and boost), giving longer series of booster vaccinations, using cytokines such as local GM-CSF and low-dose IL-2, and testing the vaccine in early-stage disease. 


\section{ACKNOWLEDGMENTS}

We gratefully acknowledge Nadine Dawkins's assistance in keeping and compiling patient research records and Debra Weingarten's help in manuscript preparation.

\section{REFERENCES}

1. Jemal A, Thomas A, Murray T, Thun M. Cancer statistics, 2002. CA Cancer J Clin 2002;52:23-47.

2. Correale P, Walmsley K, Nierod C, Zaremba S, Zhu M, Schlom $\mathrm{J}$, Tsang KY. In vitro generation of human cytotoxic $\mathrm{T}$ lymphocytes specific for peptide derived from prostate specific antigen. J Natl Cancer Inst 1997;89:293-300.

3. Correale P, Walmsley K, Zaremba S, Zhu M, Schlom J, Tsang KY. Generation of human cytolytic T lymphocyte lines directed against prostate-specific antigen (PSA) employing a PSA oligoepitope peptide. J Immunol 1998;161:3186-3194.

4. Wei C, Storozynsky E, McAdam AJ, Yeh KY, Tilton BR, Willis RA, Barth RK, Looney RJ, Lord EM, Frelinger JG. Expression of human prostate-specific antigen (PSA) in a mouse tumor cell line reduces tumorigenicity and elicits PSA-specific cytotoxic T lymphocytes. Cancer Immunol Immunother 1996;42:362-368.

5. Schlom J, Panicali D. Recombinant poxvirus vaccines. In: Rosenberg SA, editor. Biologic therapy of cancer: principles and practices. 3rd ed. Philadelphia: JB Lippincott Co; 2000. p 686-694.

6. Hodge JW, Schlom J, Donohue SJ, Tomaszewski JE, Wheeler CW, Levine BS, Gritz L, Panicali D, Kantor JA. A recombinant vaccinia virus expressing human prostate-specific antigen (PSA): safety and immunogenicity in a non-human primate. Int J Cancer 1995;63:231-237.

7. Eder JP, Kantoff PW, Roper K, Xu GX, Bubley GJ, Boyden J, Gritz L, Mazzara G, Oh WK, Arlen P, Tsang KY, Panicali D, Schlom J, Kufe DW. A phase I trial of a recombinant vaccinia virus expressing prostate-specific antigen in advanced prostate cancer. Clin Cancer Res 2000;6:1632-1638.

8. Sanda M, Smith D, Charles LG, Hwang C, Pienta KJ, Schlom J, Milenic D, Panicali D, Montie JE. Recombinant vaccinia-PSA (PROSTVAC) can induce a prostate-specific immune response in androgen-modulated human prostate cancer. Urology 1999; 53:260-266.

9. Centers for Disease Control. Vaccinia (smallpox) vaccine recommendation of the Immunization Practices Advisory Committee (ACIP). MMWR Morb Motal Wkly Rep 1991;40:1-10.

10. Arlen P, Tsang KY, Marshall JL, Chen A, Steinberg SM, Poole D, Hand PH, Schlom J, Hamilton JM. The use of a rapid ELISPOT assay to analyze peptide-specific immune responses in carcinoma patients to peptide vs. recombinant poxvirus vaccines. Cancer Immunol Immunother 2000;49:517-529.

11. Scheibenbogen C, Lee KH, Mayer S, Stevanovic S, Moebius U, Herr W, Rammensee HG, Keilholz U. A sensitive ELISPOT assay for detection of CD8 $+\mathrm{T}$ lymphocytes specific for HLA class I-binding peptide epitopes derived from influenza proteins in the blood of healthy donors and melanoma patients. Clin Cancer Res 1997;3:221-226.

12. Tsang KY, Zaremba S, Nieroda CA, Zhu MZ, Hamilton JM, Schlom J. Generation of human cytotoxic T cells specific for human carcinoembryonic antigen epitopes from patients immunized with recombinant vaccinia-CEA vaccine. J Natl Cancer Inst 1995;87:982-990.

13. McLaughlin JP, Schlom J, Kantor JA, Greiner J. Improved immunotherapy of a recombinant CEA vaccinia vaccine when given in combination with Interleukin-2. Cancer Res 1996;56: 2361-2367.

14. Hodge JW, McLaughlin JP, Kantor JA, Schlom J. Diversified prime and boost protocols using recombinant vaccinia virus and recombinant nonreplicating avian pox virus to enhance T-cell immunity and antitumor responses. Vaccine 1997;15:759-768.

15. Hodge JW, Sabzevari H, Yafal AG, Gritz L, Lorenz MGO, Schlom J. A triad of costimulatory molecules synergize to amplify T-cell activation. Cancer Res 1999;59:5800-5807.

16. Kalus RM, Kantor JA, Gritz L, Gomez Yafal A, Mazzara GP, Schlom J, Hodge JW. The use of combination vaccinia vaccines and dual-gene vaccinia vaccines to enhance antigen-specific Tcell immunity via T-cell costimulation. Vaccine 1999;17:893-903.

17. Kass E, Panicali DL, Mazzara G, Schlom J, Greiner JW. Granulocyte-macrophage colony-stimulating factor produced by recombinant avian poxviruses enriches the regional lymph nodes with antigen-presenting cells and acts as an immunoadjuvant. Cancer Res 2001;61:206-214.

18. Grosenbach DW, Barrientos JC, Schlom J, Hodge JW. Synergy of vaccine strategies to amplify antigen-specific immune responses and antitumor effects. Cancer Res 2001;61:4497-4505.

19. Murata K, Garcia-Sestre A, Tsuji M, Rodrigues M, Rodriguez D, Rodriguez JR, Nussenzweig RS, Palese P, Esteban M, Zavala F. Characterization in vivo primary and secondary CD8+ T cell responses induced by recombinant influenza and vaccinia viruses. Cell Immunol 1996;173:96-107.

20. Irvine KR, Chamberlain RS, Shulman EP, Surman DR, Rosenberg SA, Restifo NP. Enhancing the efficiency of recombinant anti-cancer vaccines with prime/boost regimens that employ 2 different vectors. J Natl Cancer Inst 1997;89:1595-1604.

21. Marshall JL, Hoyer RJ, Toomey MA, Faraguna K, Chang P, Richmond E, Pedicano JE, Gehan E, Peck RA, Arlen P, Tsang KY, Schlom J. Phase I study in advanced cancer patients of a diversified prime-and-boost vaccination protocol using recombinant vaccinia virus and recombinant nonreplicating avipox virus to elicit anti-carcinoembryonic antigen immune responses. J Clin Oncol 2000;18:3964-3973.

22. Warren TL, Weiner GJ. Uses of granulocyte-macrophage colony-stimulating factor in vaccine development. Curr Opin Hematol 2000;7:168-173.

23. Arlen PM, Gulley J, Novik L, Hamilton JM, Bastian A, Pannicali D, Palena C, Tsang KY, Schlom J, Dahut WL. A randomized phase II trial of either vaccine therapy (recombinant pox viruses expressing PSA and the B7.1 costimulatory molecule) versus hormone therapy (nilutamide) in patients (pts) with hormone refractory prostate cancer and no radiographic evidence of disease (abstract). In, Program/Proceedings of the Thirty-Eighth Annual Meeting of the American Society of Clinical Oncology, Orlando, FL: American Society of Clinical Oncology 2002;21:728a.

24. Gulley JL, Arlen PM, Bastian A, Coleman N, Hamilton M, Tsang K, Panicali D, Douglas R, Sullivan FJ, Schlom AJ, Dahut WL. A phase II study of prostate specific antigen (PSA)-based vaccine in patients with localized prostate cancer receiving standard radiotherapy (RT) (abstract). In, Program/Proceedings of the Thirty-Eighth Annual Meeting of the American Society of Clinical Oncology, Orlando, FL: American Society of Clinical Oncology 2002;21:1814a. 